\title{
Mercadotecnia, comunicación y movilización social: ciencias sin fronteras en la promoción de la salud
}

\author{
Nery Suárez Lugo \\ (Ciudad de la Habana, Cuba) \\ Escuela Nacional de Salud Pública. Ministerio de Salud Pública
}

\section{Palabras clave \\ mercadotecnia social comunicación movilización}

\begin{abstract}
Resumen
Los cambios en el pensamiento salubrista contemporáneo implican también modificaciones en toda la sociedad. Para lograrlo, se requiere aplicar estrategias que se basan en el desarrollo de las ciencias médicas y en el avance tecnológico, con el enfoque interdisciplinario de la comunicación, movilización y mercadotecnia social.

La comunicación, cuando se incluye como parte de una estrategia de mercadotecnia, enlaza en una integración adecuada con la movilización y la participación social y se potencian sus efectos. Por eso se insiste en no dividir esfuerzos en función de diversas disciplinas para abordar con una estrategia los problemas de salud, sino precisamente aunar las ciencias sociales en un enfoque integrador, que permita el cambio en la forma de concebir la salud y de lograrla, que abarque al individuo, las fuerzas públicas y la sociedad.

Diversos ejemplos presentan las posibilidades de la mercadotecnia social como estrategia apropiada en el planeamiento y ejecución de intervenciones para propiciar o modificar conductas relacionadas con la salud, ya que representa un avance sobre las estrategias tradicionales de cambio social, precisamente porque incluye la comunicación y la movilización.

Se plantea la necesidad de buscar el apoyo de la ciencia en función de preparar a los profesionales sanitarios para abordar los problemas actuales de la salud pública con el empleo de otros métodos, técnicas y herramientas que permitan lograr que del discurso se transite a la acción, de las promesas a la realidad, de la curación a la prevención, de la enfermedad a la salud y al bienestar.
\end{abstract}




\section{Marketing, communication and social mobilization: science without frontier in the promotion in health.}

\begin{tabular}{c}
\hline Keywords \\
\hline social marketing \\
communication \\
mobilization
\end{tabular}

\section{Cómo citar el artículo}

Suárez Lugo, N. (2011). Mercadotecnia, comunicación y movilización social: ciencias sin fronteras en la promoción de la salud. Revista de Comunicación y Salud, 1(1), pp. 107-118. DOI: http://doi.org/10.35669/revistadecomunicacionysalud.2011.1(1).107-118

\begin{abstract}
Changes in contemporary public health thinking also involve modifications in society as a whole. To achieve them, it is necessary to apply strategies based on the development of medical science and on technological advances, with an interdisciplinary focus on communication, mobilization and social marketing.

Communication, when included in the marketing strategy, leads to the adequate integration of social mobilization and participation and boosts its effect and impact. That is why we must insist on not dividing our efforts according to different disciplines and why we must approach healthcare problems with one integrated strategy, therefore uniting all social sciences by giving them an integrated focus which will allow changes in the way we think of health and the way we achieve it, which will include the individual, public forces and society.

Several examples present the possibilities of social marketing as an appropriate strategy to be used while planning and carrying out interventions to bring about and modify health related conducts, since it represents an improvement over traditional strategies used for social change, precisely because it includes social communication and mobilization.

Pursuing scientific support is necessary in order to teach healthcare professionals how to approach current public health problems by using other methods, techniques and tools which will allow discourse to become action, promises to become reality, treatment to become prevention and disease to become health and wellbeing
\end{abstract}

\section{Promoción de la salud: una breve historia}

Dentro de las actuales políticas de salud que se plantean a nivel mundial, probablemente la promoción de salud sea la más innovadora, amplia y posible, y a la vez la más difícil y costosa para su aplicación. Los antecedentes a este enfoque datan de muchos años atrás, pues el Informe Lalonde en 1974 ha sido considerado como el punto de partida del desarrollo moderno de la promoción de la salud, y en la Declaración de Alma Ata, en el año 1978, se señala la necesidad de actuar sobre los comportamientos, aunque centrados en la responsabilidad individual sobre la conducta y los hábitos de los individuos. 
Cinco líneas trazadas en la Carta de Ottawa desde el año 1986 incluyen en el enfoque la presencia del medio ambiente en todas sus dimensiones. Son estas la formulación de políticas públicas saludables, creación de ambientes propicios, fortalecimiento de la acción comunitaria, desarrollo de aptitudes personales y la reorientación de los servicios de salud (OMS, 1986).

Todos estos lineamientos implican un profundo cambio en lo individual, lo comunitario y lo institucional. Para lograrlo, se requiere aplicar estrategias de cambio que, de una forma u otra, tienen en su base el enfoque interdisciplinario de la comunicación, movilización y mercadotecnia social. El planteamiento anterior se ve reforzado si se consideran las tres líneas paralelas de acción que implica la promoción de salud: fomentar estilos de vida saludables, habilitar a las personas para que aumenten su capacidad de control sobre la prestación de servicios benéficos para la salud e implantar condiciones estructurales que hagan posible la salud plena y efectiva para toda la población (OPS, 2001).

Otros momentos interesantes en el discurso sobre el tema transcurren hasta la Declaración de Yakarta en 1997 sobre promoción de la salud en el siglo XXI, donde se valora ya como una inversión y se considera como un derecho humano básico e indispensable para el desarrollo social y económico. Inserta además estas categorías en un escenario donde se puedan considerar también los cambios económicos y sociales como premisas para la transformación de la salud.

En el año 2000 en México se plantea centrar la atención en demostrar la manera en que las estrategias de promoción de la salud añaden valor a la eficacia de las políticas, los programas y los proyectos sanitarios y de desarrollo, particularmente los que intentan mejorar la salud y la calidad de la vida de las personas que viven en circunstancias adversas. Es este otro avance logrado, que si bien no trasciende a la forma de actuar como se requiere, al menos sí lo hace en el pensamiento salubrista del siglo que comienza y que se traducen en la Carta de Bangkok para el fomento de la salud en un mundo globalizado en el año 2005, donde el ambiente toma una denominación más real, al hablarse de la relación entre salud y sus determinantes sociales (OMS, 2005).

Hablar de nuevas concepciones acerca de la salud y sus políticas hace reflexionar sobre nuevos paradigmas y formas de enfrentamiento, razón por la cual se impone el análisis de esas nuevas ciencias que sustentan la forma de encontrar mejoras en la situación de salud de la población.

El paradigma "más vale prevenir que tener que curar" ha sido parafraseado y no realmente asumido en las décadas que nos separan de la Declaración de Alma Ata y la Carta de Ottawa, en los escenarios donde se definen y ejecutan las políticas de salud (OPS, 2001). Prueba de ello puede obtenerse al revisar los presupuestos y recursos que se asignan a las actividades destinadas a la prevención, las estrategias programáticas, la formación de recursos humanos y la naturaleza de los servicios de salud que se ofrecen.

Asimilar nuevos paradigmas, requiere también el concurso de nuevas disciplinas de las ciencias donde convergen la mercadotecnia social, movilización social, la comunicación, así como involucrar a la comunidad mediante la participación, para lograr el cambio en cuanto 
a los paradigmas tradicionales de ver la salud relacionada con la curación de la enfermedad.

No resultó posible enfrentar el paradigma de la salud vista como un reto para reducir iniquidades, tal como se expresara en el propósito de "Salud para Todos", ampliar el alcance de la prevención, y ayudar a la población a enfrentarse a sus circunstancias, sin fomentar la participación social, fortalecer los servicios comunitarios de salud y promover políticas públicas saludables, sin auxiliarse de las ciencias de la comunicación, la mercadotecnia y la movilización social (Suárez, 2004). Hoy, los Objetivos de Desarrollo del Milenio corren el riesgo de no lograr su propósito si no se hace uso de las herramientas que ofrecen las ciencias sociales y del comportamiento (OMS, 2004).

El planteamiento de Hiroshi Nakajima en 1988 de ver la salud como producto de la acción social y no como un resultado de la atención médica, sentó precedentes para enfocar la salud con ese nuevo paradigma, pero indudablemente que unido a la necesidad de expresarse en una voluntad política, también requería enfrentarlo con el apoyo de esas disciplinas. Es obvio que estos denominados nuevos paradigmas se basan en el desarrollo de las ciencias médicas y en el avance tecnológico, que han permitido analizar la situación epidemiológica a nivel mundial y han llevado a la clara definición de que resulta necesario modificar estilos de vida y enseñar a la población a asumir conductas que tengan un impacto positivo en su salud, si se quiere modificar el actual cuadro epidemiológico (Starfield et al., 2008; González Pérez, 2002). Las muertes prevenibles, tanto por responsabilidad individual como por responsabilidad pública, sustentan el empeño de educar, informar, persuadir, entrenar, modificar el medio ambiente y adecuar las condiciones en que vive el hombre, para que pueda obtener una mayor calidad de vida y, por ende, una mejor salud. Esta aseveración permite resumir el desarrollo del pensamiento sobre la promoción de la salud desde el último cuarto del siglo pasado hasta el momento actual (Jenkins, 2005).

Pero transitar del discurso a la acción requiere en primer término la decisión de actuar con tal propósito y luego el empleo, entre otros, de programas de mercadotecnia social, con estrategias de comunicación, movilización social y participación comunitaria. Se trata de nuevos enfoques, nuevas estrategias, nuevos métodos y nuevas técnicas para facilitar la acción individual y social. Solamente de esta forma será posible acortar la distancia entre la formulación de políticas y la realidad, que en términos de salud se puede traducir en vocablos que se sienten muy fuertes, pero que son realidad: la vida o la muerte; de lo contrario, la meta trazada de "Salud para Todos" hubiese sido alcanzada en el año 2000 y no sería en la actualidad un propósito y una esperanza por los que seguir trabajando en el futuro, sino una realidad del presente.

\section{Mercadotecnia, movilización y comunicación social}

La aceptación en la década del 60 del siglo XX de la mercadotecnia social como estrategia de cambio de conductas, entre las que se encuentran las que tienen que ver con la salud, sitúan a la vez la comunicación en un plano central, considerándola uno de los componentes esenciales de todo programa de cambio y haciendo necesario que los profesionales de la salud se involucren en estos procesos y se capaciten en técnicas y métodos de comunicación social (Suárez, 2004). 
La concepción de la promoción de salud como elemento clave para transformar la situación de salud de la población, hace énfasis en ambas ciencias, aunque no siempre de forma explícita y, además, acude a la movilización social como vía para involucrar a la comunidad en las decisiones que tienen que ver con las posibilidades de acceder a un estilo y condiciones de vida sanas.

La participación comunitaria como concepto socialmente aceptado entre los salubristas de estos tiempos, implica a la vez la necesidad de conocer las técnicas y métodos que posibilitan tanto la comunicación como la mercadotecnia y la movilización, pues permiten el cambio en la forma de pensar de la población y los decisores de las políticas públicas, para enfrentar la salud como un fenómeno social, que requiere, en primer término, del principio de equidad en su acceso, en los factores que la determinan y en los que se requiere asumir la responsabilidad social que les corresponde a cada uno.

La movilización social se desarrolló a partir del interés del Fondo de Naciones Unidas para la Infancia (UNICEF) en la participación comunitaria como un ingrediente esencial para el servicio de distribución a los sectores más pobres de la población urbana y rural (Fundación W. K. Kellogg, 2001). La diferencia esencial radica en que la participación comunitaria restringe la comunidad al nivel primario, los beneficiarios, mientras la movilización social le da un más amplio enfoque a la comunidad, incluyendo todos los posibles sectores y niveles de la sociedad (Sanabria, 2004).

El concepto probablemente fue sintetizado de una gama de experiencias, pero ganó estatura y especificidad en el altamente exitoso programa expandido de inmunización (EPI), el cual requirió de un cambio en los enfoques tradicionales, para obtener resultados y en el que se empleara una estrategia de mercadotecnia social con la utilización de un adecuado programa de comunicación que incluye la movilización para lograr la participación comunitaria. Otros ejemplos pueden demostrar la utilidad de este enfoque, entre ellos se puede hacer referencia a los programas de planificación familiar, nutrición, abuso de alcohol y drogas y de tabaquismo, por citar algunos de los más conocidos.

La aceptación de las cuatro $p$ del marketing en la mercadotecnia social, en las que se habla de un producto social, que puede ser un objeto tangible, una idea o una práctica, con un precio monetario o visto como una oportunidad o valor físico o psicológico, colocan la comunicación social en un lugar prioritario, donde no solo los mensajes constituyen la p de la promoción para dar a conocer todo lo relacionado con el producto, sino que en múltiples ocasiones el lugar o punto de distribución son los propios canales de comunicación.

La segmentación del mercado constituye a la vez una información esencial para definir no solamente el producto social que se va a promover, sino la estrategia de comunicación para cada grupo meta de acuerdo a la segmentación de mercado realizada, para que posibilite el posicionamiento del mismo, ya sea una idea, una práctica y tenga o no asociado un objeto tangible. Este análisis permite conocer el importante papel que desempeña la comunicación en la formación y cambio de conductas y en cuanto está sujeta la aceptación del producto social que se va a promover a una adecuada mezcla de mercadotecnia, en la que sin subestimar el papel del diseño, la definición del precio y los puntos de distribución, se evidencia la importancia de un adecuado programa de comunicación social. 
La literatura sobre mercadotecnia social incluye en uno de los aspectos que se deben considerar, la movilización social de los denominados grupos de influencia en relación con el cambio que se quiere lograr. Los aliados, opositores y neutros, son los grupos que se clasifican en función de su adhesión o no a la causa social o producto social que se va a promover. La comunicación incluye en estos casos la denominada advocacy, cuya mejor traducción puede ser la abogacía, el lobbing, utilización de embajadores de buena voluntad y el uso de los medios de comunicación social. Pueden considerarse además como formas de movilización, la gubernamental, comunitaria, corporativa y de beneficiarios, en las cuales se utilizan los programas de entrenamiento, técnicas participativas, medios masivos de comunicación, entre otros. Aunque no siempre tiene el mismo grado de importancia, la movilización social es una estrategia muy útil para favorecer los cambios sociales si se integra de forma adecuada con la estrategia de mercadotecnia social (Suárez, 2007). Hay programas de cambio social, específicamente relacionados con conductas de salud, que requieren más de ello. Por ejemplo, mientras que en un programa de prevención y control del tabaquismo es más importante considerar los grupos de influencia y actuar sobre ellos, cuando se trata de un programa de calidad del agua se requiere mayor participación gubernamental y comunitaria.

La movilización social es un proceso autosustentable, un acercamiento a distintos niveles, tanto de abajo a arriba como de arriba a abajo, basado en un entendimiento profundo y comprensivo del contexto social, cultural, político y económico de un lugar o país, cuidadosamente planificado y costeado desde el punto de vista de la factibilidad, para asegurar que la demanda creada en el proceso de movilización sea lograda. Es también una programación intensiva en los puntos de presión seleccionados y un proceso dinámico que requiere una ágil respuesta de soporte.

Con la movilización social, se aspira a incrementar la conciencia de las poblaciones, el conocimiento y la habilidad para organizar el autoaseguramiento, ayudar a la gente a estar motivada, conocer sus derechos y deberes, así como reclamar la satisfacción de sus necesidades, a comprender y modificar las ideas y creencias y a movilizar todos los recursos disponibles.

La movilización social, por lo tanto, es un acercamiento de creación de demanda que persigue atraer la participación total de la comunidad o nación y la pertenencia a un programa. Es el proceso de conciliar a todos los posibles y prácticos aliados sociales, intersectoriales, para aumentar la percepción y por tanto la demanda respecto de un determinado programa de desarrollo, para ayudar en la provisión de recursos y servicios, y para fortalecer la participación comunitaria en la sostenibilidad y la autoconfianza. Es también la concertación voluntaria, intensa y sostenida de los recursos y esfuerzos de las instituciones sociales claves en respaldo y provecho de un programa de acción de alta prioridad y claro beneficio para la mayoría de los ciudadanos de un país.

Es importante considerar la presencia de formas de participación de la sociedad en la toma de decisiones, la implementación, el beneficio y la evaluación. La participación comunitaria se logra mediante la movilización social y es el proceso de educación y habilitación por el cual la gente se asocia con aquellos que tienen capacidad para atenderla. Identifica problemas y necesidades, y asume por sí misma las responsabilidades de planificar, manejar, controlar y evaluar las acciones colectivas que resulten necesarias. Es la forma de hacer protagonistas 
a los que de una forma u otra son beneficiarios de las acciones y servicios de salud (Álvarez, Sanabria et al., 1996).

La comunicación se convirtió rápidamente en una ciencia y sus profesionales se involucran en equipos multidisciplinarios de especialistas en sociología, psicología, antropología, entre otros. Cada vez, toma mayor importancia el impacto de un minuto de comunicación en la radio y la televisión, o un pequeño espacio en la prensa. El desarrollo de la ciencia y los procesos de democratización y participación de la sociedad civil hacen que igual que en políticas de salud, nuevos paradigmas soporten hoy en día la comunicación, cobrando cada vez más importancia el papel de las nuevas tecnologías de información, unidas a factores puramente sociales como son la identidad cultural.

Las estrategias tradicionales de comunicación para la promoción de la salud comprenden tres aspectos esenciales: abogacía, movilización social y participación comunitaria. De esta forma se va comprobando cómo se entrelazan las ciencias sociales, que en estrecha relación permitirán dar respuesta a los actuales paradigmas de las políticas de salud de estos tiempos. Sin embargo, hacer uso de estrategias de mercadotecnia social para integrarlas, constituye una asignatura aún pendiente en la mayoría de los proyectos.

La comunicación social en salud es vista como un proceso de influencia social multitudinario, que proporciona conocimientos, forja actitudes y provoca prácticas requeridas en la población por los programas de salud para mejorar el estado de esta última. Es un sistema de intervenciones para la modelación masiva de conductas propicias al buen estado de salud y también el intento sistemático de influir positivamente en las prácticas de salud de poblaciones extensas, utilizando principios y métodos de la comunicación masiva, diseño de la instrucción, la mercadotecnia social, análisis del comportamiento y antropología médica (Mckee, 1992).

Uno de los aspectos más debatidos en relación con la mercadotecnia, comunicación y movilización social es lo referente a la manipulación, esto es, la ética de su empleo, es por eso que resulta necesario traer el tema del denominado dilema al respecto.

También es importante reconocer el papel que desempeña la comunicación y la información en los programas de cambio (Suárez, 2004) y cómo la ética también se encuentra presente en la forma en que se concibe, cómo se maneja la comunicación en aras de llegar a los receptores, destinatarios y grupos meta, siempre con el principio de que se informa y persuade para que decidan qué es lo que quieren hacer.

La manipulación y la publicidad engañosa son puntos de serio debate en los organismos internacionales que velan por el respeto a los derechos ciudadanos y que están muy al tanto de cómo las transnacionales, cada vez más buscan las formas de llegar al consumidor sin información y desprotegido, para reemplazar sus mercados con aquellos que, o bien ya han tomado decisiones consecuentes con su salud y su calidad de vida, o bien han muerto producto de no haber podido disponer de los conocimientos requeridos para hacer un balance adecuado entre lo que debo y no debo consumir. 


\section{Relaciones sin fronteras}

Visto de esta forma, es importante considerar que la comunicación para la salud resulta insuficiente para dar respuesta al paradigma actual, mientras que se potencian sus efectos cuando se incluye como parte de una estrategia de mercadotecnia y se enlaza en una integración adecuada con la movilización y la participación social.

No se trata de dividir esfuerzos en función de diversas disciplinas para abordar con una estrategia los problemas de salud, sino precisamente de aunar las disciplinas de las ciencias sociales en un enfoque integrador, que permita el cambio en la forma de concebir la salud y de lograrla, que abarque al individuo, las fuerzas públicas y la sociedad (Suárez, 2008).

La mercadotecnia social es una respuesta para los cambios que se requieren, porque considera el producto social que se va a promover incluyendo los mismos elementos que plantea la venta de productos, y para que sea adoptado a nivel poblacional, se plantea una estrategia que abarca todos los planos en los que resulta necesario hacer la transformación para que se produzca la adopción.

Resulta muy interesante el análisis que sobre la mercadotecnia social, la promoción de salud y la comunicación, realizan Hastings y Haywood (1994), del Center for Social Marketing de la Universidad de Strathclyde, de Glasgow, en el Reino Unido, y Buchanan, Sasiragha y Zafar, (1994) de la Universidad de Massachusetts, en Estados Unidos. El punto de vista, que comparto, de estos autores es que en ningún momento la mercadotecnia social reemplaza los conceptos de promoción de salud, sino que ambos tienen que desarrollarse paralelamente. Adoptar posiciones respecto a cuál de las disciplinas es más antigua resulta una postura anticientífica y que detiene el progreso.

La mercadotecnia social es la aplicación de los principios de la mercadotecnia a programas sociales. Es un proceso social que implica el desarrollo de programas para incrementar la aceptación de ideas o prácticas sociales en un grupo diana, y por ello es utilizado de forma sistemática en la solución de problemas relacionados con la salud, la promoción de adopción de conductas saludables, así como de utilización adecuada y racional de los servicios de salud. Se ha comprobado que también la mercadotecnia social es una estrategia apropiada para el planeamiento y ejecución de intervenciones que tienen el propósito de propiciar o modificar conductas relacionadas con la salud, y representa un avance sobre las estrategias tradicionales de cambio social.

La mercadotecnia social le aporta a la promoción de salud más efectividad ya que está sustentado científicamente que información, educación y comunicación, por sí solas, no resultan suficientes para lograr los cambios requeridos en el comportamiento de las personas. Sin embargo, como unión de métodos y técnicas de diversas disciplinas crea sinergia y potencia los efectos aislados, permitiendo los cambios con mayor rapidez y haciendo los programas de salud menos costosos.

Se incluye en este análisis la disposición de la sociedad para aceptar el cambio, pues es a partir de que esto sucede que se podrán gestar políticas públicas que tributen a la salud y al 
bienestar (Suárez, 2004). Un ejemplo muy actual lo constituyen los esfuerzos que la Organización Mundial de la Salud (OMS) y la Oficina Sanitaria Panamericana (OPS) han realizado para combatir la epidemia tabáquica a nivel mundial. Luego de esfuerzos en la educación para concienciar a la población sobre la nocividad de fumar y no obtener resultados, se logra aprobar el Convenio Marco para el Control del Tabaco (CMCT), que representa una herramienta fundamental para frenar la epidemia del tabaquismo para los gobiernos y la sociedad civil. Los gobiernos, porque a partir del mismo pueden promulgar políticas públicas para reducir la accesibilidad fundamentalmente y la sociedad civil puede contribuir especialmente logrando involucrar a los tomadores de decisiones y líderes de opinión, empoderando a los no fumadores en su derecho a respirar aire puro y cómo ejercerlo, así como formar redes de organizaciones que puedan facilitar el uso de los instrumentos legislativos existentes (OMS, 2003).

Este documento, el de mayor rango de que dispone la salud pública hasta la fecha en cuanto a conciliar acciones de prevención y promoción de la salud a nivel mundial, supera a esfuerzos anteriores porque precisamente se apropia no solo de la comunicación, sino que busca con la participación y la movilización social transformar el ambiente para que este resulte coherente con el propósito de reducir el tabaquismo, al propiciar barreras al consumo, no solo mediante el precio y la comercialización, sino también con la prohibición de fumar en lugares públicos.

Las barreras generalmente requieren del apoyo de la legislación, al menos mientras no se logre el posicionamiento de la idea y la práctica que se promueve (Suárez, Caraballoso y Rodríguez, 2008). En el caso que se analiza, mientras que la aceptación social del tabaquismo se mantenga no se puede dejar a la espontaneidad la protección al no fumador, fundamentalmente a los niños y jóvenes, y las normativas legales constituyen el punto de apoyo, no solo para la protección a la salud, sino precisamente para actuar en función de la no aceptación social y de esa manera lograr esa disposición para la aprobación del cambio a nivel de la sociedad que requiere la salud pública.

La falta de información sobre las posibilidades que ofrece la mercadotecnia social para los programas de cambio de comportamiento en las relaciones sexuales es también un denominador común cuando se valoran los lentos efectos de los programas de prevención y control de las ITS y del VIH/sida. Sin embargo, los progresos se hacen sentir y ya se abordan con estas herramientas en algunos de los programas auspiciados por organismos internacionales, aunque sea de forma discreta. Los problemas parecen concentrarse más que en el diseño de las estrategias en la ejecución y desarrollo, fundamentalmente por la falta de conocimientos y habilidades de ponerlas en práctica y no tomar en consideración la investigación de mercados que acompañe todo el proceso de toma de decisiones para producir los resultados necesarios.

Todas las epidemias no pueden ser abordadas con estrategias similares y todos los escenarios tienen sus particularidades. La mercadotecnia social ofrece alternativas de estrategias diferentes no sólo por ser públicos diferentes, sino por abordar problemáticas o manifestaciones distintas de un mismo problema. La experiencia ha demostrado, que aunque existan estrategias globales porque el $\mathrm{VIH} /$ sida constituye un problema global, cada país tiene que enfrentarlo desde su contexto, la magnitud de la epidemia y a partir de investigaciones particulares de su población y su medio externo. 
Una experiencia interesante en Cuba constituyó el empleo de una estrategia de mercadotecnia social incluyendo la movilización y la comunicación social como parte del programa "Fortalecimiento de la respuesta nacional multisectorial para la prevención y atención de la epidemia del VIH/sida en la República de Cuba" auspiciado por el Fondo Mundial de lucha contra el sida, la malaria y la tuberculosis.

Este programa, se sustenta en la movilización social y emplea la mercadotecnia social con un fuerte énfasis en la comunicación, con el propósito fundamental de lograr que las relaciones sexuales sean cada vez más protegidas para lograr que se frene la epidemia. La estrategia de mercadotecnia social a emplear para lograr relaciones sexuales protegidas se centra en promover el uso del condón masculino y pretende lograr el posicionamiento de una idea y una práctica: conducta sexual protegida, con un producto tangible, el condón. Se resume entonces en propiciar la adquisición y uso al tener disponibilidad de condones masculinos de alta calidad, distribuidos de manera que garanticen la accesibilidad tanto geográfica, en lugares, horarios, como económica por precios accesibles, informando y educando sobre las ventajas de una relación sexual protegida, para lograr que la población tome conciencia que permita desmitificar los elementos psicológicos y sociales asociados y propiciar el uso correcto del condón.

La evaluación del programa en Cuba puso de manifiesto la utilidad de la mercadotecnia al insertarse como estrategia y a la vez la necesidad de que la investigación acompañe todo el proceso para producir los resultados necesarios (Suárez, 2007).

\section{Conclusiones}

El déficit en la capacidad de interpretación de los fenómenos y problemas sociales trae como consecuencia la carencia del pensamiento prospectivo que sustente el paradigma preventivo y de promoción de la salud para que finalmente desplace al curativo-asistencial. Generar cambios sustantivos que den respuesta a las demandas esenciales del desarrollo humano requiere transformaciones en la forma de pensar de los decisores de las políticas públicas para resolver los problemas del presente y ejercer acciones que permitan en el futuro mejorar el estado de salud de la población.

La mercadotecnia, en su enfoque social, al integrar la comunicación, la movilización de los diferentes sectores de la sociedad y la participación de la comunidad, facilita realizar acciones de promoción de la salud con una base científica y una estrategia adecuada, al ofrecer el instrumental para el cambio de conductas para promover la salud, el desarrollo social y ambiental. Se trata de encontrar el apoyo de la ciencia, de preparar a los profesionales sanitarios para abordar los problemas actuales de la salud pública que requieren de nuevas competencias y herramientas, del empleo de otros métodos y técnicas para lograr que del discurso se transite a la acción, de las promesas a la realidad, de la curación a la prevención, de la enfermedad a la salud y al bienestar. 
Referencias

Álvarez Reyes, Luisa; Sanabria Ramos, Giselda, et al. (1996). Metodología para la caracterización de la participación comunitaria en salud. Revista Cubana Salud Pública. Vol. 22, n 1 , pp. 85-101.

Buchanan, David; Sasiragha, Reddy y Zafar, Hosain. (1994). Social Marketing: A Critical Appraisal. Health Promotion Internacional. Vol 9, n 1, pp. 49-57.

Fundación W. K. Kellogg. (2001) [1989]. Manual de comunicación social para la promoción de la salud en adolescentes. Washington DC: Oficina Sanitaria Panamericana. Organización Mundial de la Salud.

González Pérez, Ubaldo. (2002). El concepto de calidad de vida y la evolución de los paradigmas de las ciencias de la salud. Revista Cubana Salud Pública. Vol. 28, n² 2, pp. 157-175.

Hastings, Gerard y Haywood, Amanda. (1994) Social Marketing: A Critical Response. Health Promotion Internacional. Vol 69, n 1, pp. 59-63.

Jenkins, C. David. (2005). Mejoremos la salud a todas las edades. Un manual para el cambio de comportamiento. Washington DC: Organización Panamericana de la Salud. Publicación científica y técnica $\mathrm{n}^{\circ} 590$.

Mckee, Neill. 1992 [1982]. Social Communication and Social Marketing in Developing Communities. Chicago: University of Chicago Press. Disponible en http://www.bibliotecavirtual.clacso.org.ar/ar/libros/edicion/media/ [recuperado el 23 de febrero de 2010].

Oficina Sanitaria Panamericana. Organización Mundial de la Salud. (2001). Mensaje del Director. En Informe Anual del Director. Promoción de la Salud en las Américas. Washington DC: Oficina Sanitaria Panamericana. Organización Mundial de la Salud. Disponible en http://www.paho.org/Spanish/D/DO302_prelim.pdf [Recuperado el 23 de febrero de 2010].

Organización Mundial de la Salud. (1986). Carta de Ottawa para la Promoción de la Salud. Conferencia Internacional auspiciada por la OMS y la Asociación Canadiense de Salud Pública. Toronto, Canadá: Organización Mundial de la Salud. Disponible en http//www.wilkipedia.org/wilki/Carta_de_Otawa. [Recuperado el 23 de febrero de 2010].

Organización Mundial de la Salud. (2005). Carta de Bangkok. VI Conferencia Mundial de Promoción de Salud. Ginebra: Organización Mundial de la Salud. [Serie en Internet]. Disponible en http://www.who.int/healthpromotion/conferences/6gchp/BCHP_es.pdf [Recuperado el 23 de febrero de 2010].

Organización Mundial de la Salud. (2004) Objetivos de Desarrollo del Milenio relacionados con Salud. Ginebra: Organización Mundial de la Salud. Disponible en: http://www.who.int/mdg/es/ [Recuperado el 23 de febrero de 2010].

Organización Mundial de la Salud. (2003). Convenio Marco para el Control del Tabaco. Ginebra: Organización Mundial de la Salud, WHA 56.1. Disponible en http://www.paho.org/Spanish/DD/PUB/sa56r1.pdf [Recuperado el 02/04/10].

Sanabria Ramos, Giselda. (2004) Participación social en el campo de la salud. Revista Cubana de Salud Pública. Vol. 30, n 3, pp. 1-11. 
Suárez Lugo, Nery. (2004) [2001]. Marketing en salud: una perspectiva teórico-práctica. Colombia: Editorial CATORSE.

Suárez Lugo, Nery. (2007). Enfoque social de la mercadotecnia sanitaria. La Habana: Editorial Ciencias Médicas.

Suárez Lugo, Nery. (2008). Mercadotecnia en el Sistema Nacional de Salud de Cuba. 2005. Revista Cubana de Salud Pública. Vol. 34, n 1, pp. 1-11.

Suárez Lugo, Nery. (2004). Tabaco o salud: una decisión social. Revista Horizontes Sanitarios. Vol. 3, $\mathrm{n}^{\circ}$ 2, pp. 12-21.

Suárez Lugo, Nery; Caraballoso Hernández, Magaly y Rodríguez Cuélez, Delvis. (2008). Legislación para el control del tabaquismo en Cuba. Revista Cubana Salud Pública. Vol. 34, ${ }^{\circ}$ 3.

Suárez Lugo, Nery. (2007). Evaluación de la Estrategia de Mercadotecnia Social del Condón en Cuba. Revista Cubana de Salud Pública. Vol. 33, n 1, pp. 1-9.

Starfield, Barbara.; Hyde, John.; Gérvas, Juan. y Heath, lona. (2008). The Concept of Prevention: A Good Idea Gone Astray. Journal of Epidemiology and Community Health. Vol. 62, $n^{\circ} 7$, pp. 580-583. 